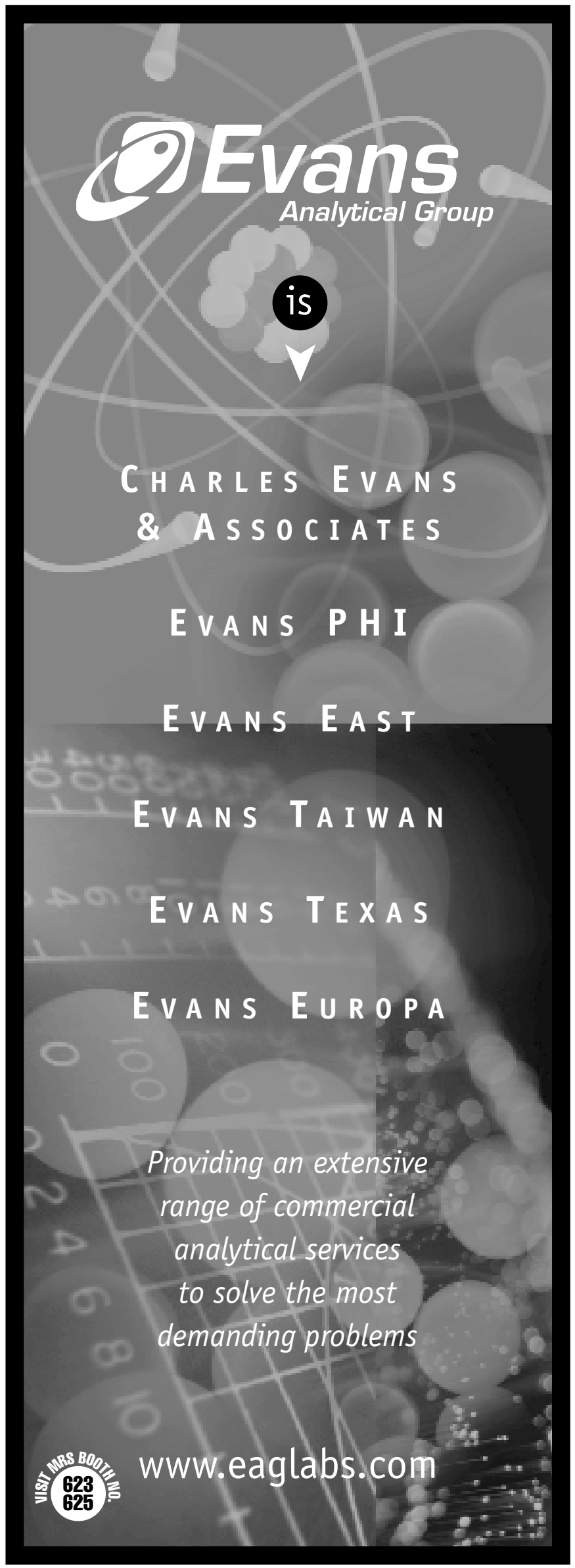

Circle No. 16 on Inside Back Cover several small $I_{n}$ clusters exhibit unexpected dynamic behavior and could dominate the precipitation of I's under conditions of supersaturation. In the February 12 issue of Physical Review Letters, S.K. Estreicher and M. Gharaibeh of Texas Tech University, P.A. Fedders of Washington University, and Pablo Ordejón of Institut de Ciència de Materials de Barcelona report that $a b$ initio molecular-dynamics (MD) simulations show that the di-interstitial $\left(\mathrm{I}_{2}\right)$ clusters and one particular tri-interstitial $\left(\mathrm{I}_{3} \mathrm{a}\right)$ cluster diffuse remarkably fast, too fast to be seen experimentally.

$A b$ initio MD simulations with pseudo-atomic basis sets (SIESTA) were performed in periodic supercells containing 64 (with four $k$-points), 128 (one $k$-point), and 216 (one $k$-point) host atoms. The basis sets varied from minimal (one set of $s$ and $p$ orbitals per $\mathrm{Si}$ atom) to double-zeta-plus polarization (two sets of $s$ and $p$ orbitals plus one set of $d$ orbitals per Si atom). The electronic energy was obtained using density-functional theory within the local density approximation. The exchange-correlation potential was that of Ceperley-Adler as parameterized by S. Perdew and A. Zunger. Norm-conserving pseudopotentials in the Kleinman-Bylander form were used to remove the core electrons from the calculations. A time step of $2.0 \mathrm{fs}$ was used in all the simulations.

After simulated quenching starting from many possible initial configurations, the researchers determined that only one configuration of $\mathrm{I}_{2}$ and two configurations of $\mathrm{I}_{3}$ are stable. Several structures for $\mathrm{I}_{4}$ were also obtained. Constant-temperature MD simulations at $1000 \mathrm{~K}$ and $77 \mathrm{~K}$ show that $\mathrm{I}$ and $\mathrm{I}_{4}$ behaved as expected, vibrating around their equilibrium sites. In the metastable $\mathrm{I}_{3}{ }^{\mathrm{b}}$ cluster, the three self-interstitials continuously exchange positions without motion of the center of mass of the defect. However, $\mathrm{I}_{2}$ and $\mathrm{I}_{3}{ }^{\mathrm{a}}$ diffuse with remarkable ease. Both clusters are centered around a single bond-centered site and two (for $\mathrm{I}_{2}$ ) or three (for $\mathrm{I}_{3}$ ) I's join forces to displace the same host atom, thus facilitating the exchange process and allowing fast diffusion.

DONALD F. CARTER

\section{Aluminum Induces Crystallization in Amorphous Silicon at $150^{\circ} \mathrm{C}$}

Aluminum-induced crystallization (AIC) has been observed in amorphous silicon $(\alpha-\mathrm{Si}: \mathrm{H})$ films. As reported in the February issue of Electrochemical and Solid-State Letters, researchers at the University of Arkansas deposited $\mathrm{Al}$ onto $\alpha$ $\mathrm{Si}: \mathrm{H}$ films and annealed them. Transmission electron microscopy (TEM) and x-ray diffraction (XRD) showed that crystallization occurred at $150^{\circ} \mathrm{C}$ and above. No further crystallization occurred after the $\mathrm{Al}$ was removed.

The $\alpha$-Si:H films were deposited by ultrahigh-vacuum plasma-enhanced chemical vapor deposition (PECVD) onto carbon-coated nickel grids. A 50-nm-thick Al film was then deposited by vacuum evaporation. Next, the films were annealed at temperatures between $100^{\circ} \mathrm{C}$ and $250^{\circ} \mathrm{C}$ under 2 mTorr vacuum. The $\mathrm{Al}$ was removed by $\mathrm{KOH}$ etching.

TEM of a film annealed at $140^{\circ} \mathrm{C}$ for 20 min showed no large nucleation sites. The electron diffraction pattern (EDP) shows broad $\mathrm{Si}$ halos indicative of amorphous material. TEM of a film annealed at $150^{\circ} \mathrm{C}$ for $30 \mathrm{~min}$, however, shows the film has completely crystallized into randomly oriented polycrystalline silicon, with grain sizes ranging from 0.2 to $0.5 \mu \mathrm{m}$. The EDP clearly shows the $\langle 111\rangle,\langle 220\rangle$, and $\langle 311\rangle$ rings. At lower magnification, the micrograph shows a honeycombnetwork morphology, which may indicate that the growth pattern of the polycrystalline silicon was dendritic. Similar features have previously been observed by other researchers. 
For the XRD measurements, 400-nmthick $\mathrm{Al}$ was deposited onto 500 -nm thick PECVD $\alpha-S i: H$. The area of the Si $\langle 111\rangle$ peak was measured at different temperatures. At $140^{\circ} \mathrm{C}$, there was no indication of crystallization. After $2-3 \mathrm{~min}$ at $150^{\circ} \mathrm{C}$, the area of the peak began to increase. More than $40 \%$ of the film crystallized within $15 \mathrm{~min}$. The $\mathrm{Al}$ was removed by etching and the XRD measurements were repeated, with temperatures up to $250^{\circ} \mathrm{C}$. No increase in the $\langle 111\rangle$ peak area was observed, implying that no crystallization occurred after the Al film was removed, even when annealed at $250^{\circ} \mathrm{C}$ for $20 \mathrm{~min}$.

Since Al diffusion in $\alpha-\mathrm{Si}: \mathrm{H}$ at $150^{\circ} \mathrm{C}$ has been previously reported by several scientists, these researchers believe that the activation energy for crystallization was reduced by $\mathrm{Al}$ atoms in the $\alpha-\mathrm{Si}: \mathrm{H}$. This agrees with previous work by other researchers showing that implanting $\mathrm{P}, \mathrm{B}$, or As in $\alpha-\mathrm{Si}: \mathrm{H}$ enhances the solid-phase crystallization.

\section{ELIZABETH SHACK}

\section{High-Quality, Manganese-Doped ZnSe Nanocrystals Prepared by High-Temperature Organometallic Synthesis}

A team of researchers has unveiled a method of preparing manganese-doped ZnSe nanocrystals that are not only superior to previously doped II-IV materials, but are also comparable in quality with the best-known undoped nanocrystals. In their work, published in the January issue of Nano Letters, D.J. Norris (NEC Research Institute), N. Yao (Princeton University), and F.T. Charnock and T.A. Kennedy of the U.S. Naval Research Laboratory have used an adapted version of the high-temperature, organometallic synthesis method developed by Hines and Guyot-Sionnest in 1998. Instead of the previously used organometallic source for Mn (diethylmanganese), the researchers used dimethylmanganese, whose lifetime is significantly longer.

To verify that Mn was actually embedded inside the ZnSe nanoparticles, instead of segregating to their surfaces as in the case of CdSe, the researchers applied optical, magnetic circular dichroism (MCD) and electron paramagnetic resonance (EPR) measurements. While photoluminescence and photoluminescence-excitation spectra showed successful doping, they could not guarantee that $\mathrm{Mn}$ was actually substituting for $\mathrm{Zn}$ in the nanocrystals. The confirmation for that came from the EPR results, which have shown values similar to those for Mn located at cubic lattice sites in bulk ZnSe. In addition to providing evidence for successful doping, the MCD data also provided clues about the doping level in the samples prepared, with values within the limit of one manganese atom per crystallite or less, implying an atomic Mn:Zn ratio of $0.025-0.125 \%$.

This simple method for preparing and testing high-quality ZnSe nanocrystals could provide a model for gaining a detailed understanding of dilute magnetic semiconductor nanocrystals and their potential applications such as trapped single-spin manipulation for the developing field of spintronics.

Claudiu Muntele

\section{Reversible Thermal Denaturation of Immobilized Met-Hemoglobin Demonstrated}

Enzymes, which have been extremely desirable catalysts for chemical reactions, are confined to use at room temperature because of their thermal instability. Immobilized met-hemoglobin (Fe(III) $\mathrm{Hb}$ ), which showed peroxidase activity in the presence of hydrogen peroxide, is one such example. It starts to denature at $73^{\circ} \mathrm{C}$ with the loss of peroxidase activity and $\alpha$-helical content. In the February issue of Chemistry of Materials, professor C.V. Kumar and coworker A. Chaudhari from the University of Connecticut report that $\mathrm{Hb}$, immobilized at the galleries of $\alpha$-zirconium carboxymethyl phosphonate $(\alpha-\mathrm{ZrCMP})$, did recover after heat denaturation.

The researchers used x-ray diffraction and spectral measurements of circular dichroism and Fourier transform infrared (FTIR) absorbance to verify the reversible thermal denaturation of immobilized $\mathrm{Hb} / \alpha-\mathrm{ZrCMP}$. The increased $\mathrm{d}$ spacings of the immobilized $\mathrm{Hb}$ on a series of galleries shrank upon heating; however, the $\mathrm{d}$ spacings of $\mathrm{Hb} / \alpha-\mathrm{ZrCMP}$ recovered after cooling for an hour while the others did not, even after cooling for an extended period. Also, the native protein had characteristic bands in its circular dichroism spectra and IR absorption spectra. The characteristic bands of renatured $\mathrm{Hb} / \alpha-\mathrm{ZrCMP}$ remained in position or nearly completely recovered after cooling. However, those characteristic bands either shifted or only partially recovered for other immobilized $\mathrm{Hb}$ after denaturation and cooling. The peroxidase activity of immobilized $\mathrm{Hb} / \alpha-\mathrm{ZrCMP}$ after denaturation gradually recovered and reached

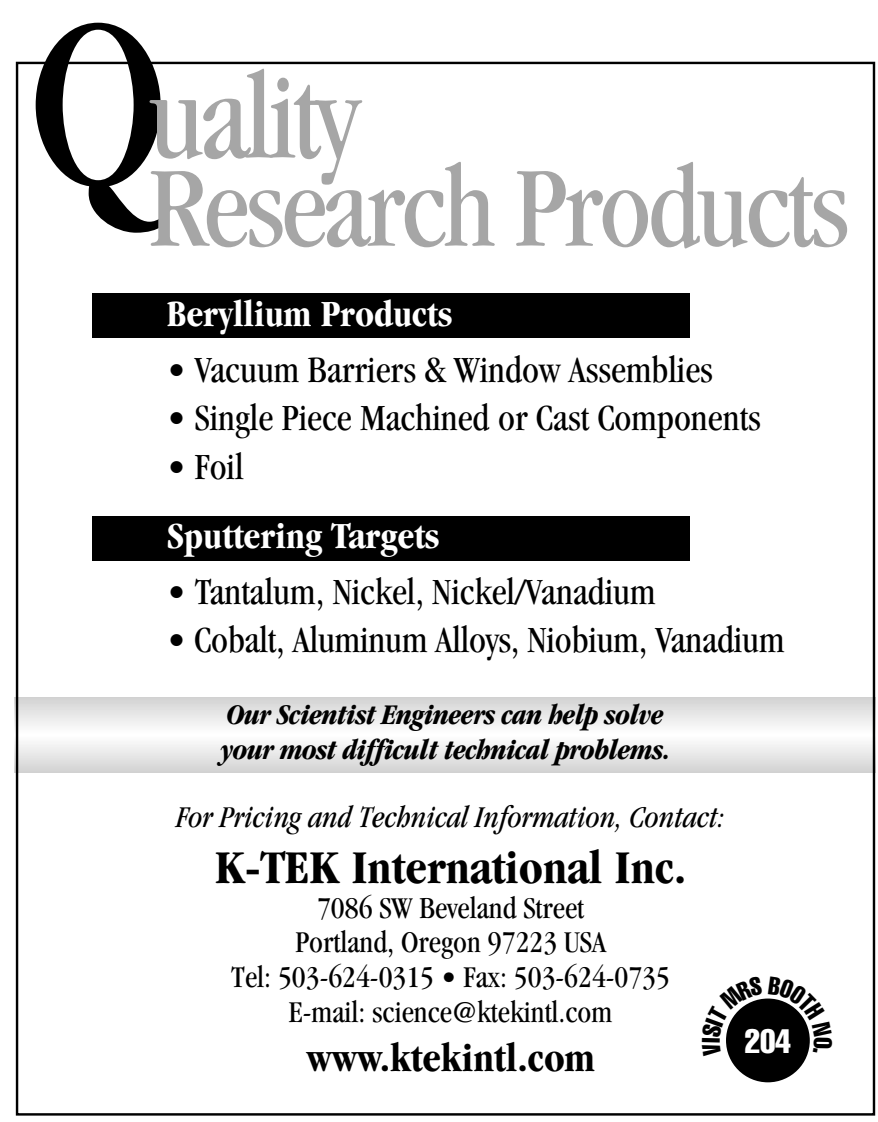

Circle No. 19 on Inside Back Cover 